\title{
E-CLOUD, the open microgrid in existing network infrastructure
}

\author{
David Vangulick \\ ORES \& University of Liège - Belgium \\ david.vangulick@ores.net \\ Olivier Devolder \\ N-SIDE - Belgium \\ ode@n-side.com
}

\author{
Bertrand Cornélusse \\ University of Liège - Belgium \\ bertrand.cornelusse@ulg.ac.be
}

\author{
Thierry Vanherck \\ University of Mons - Belgium \\ Thierry.vanherck@umons.ac.be \\ Simon Lachi \\ RESA - Belgium \\ simon.lachi@resa.be
}

\begin{abstract}
The main goal of the E-Cloud, as with every microgrid, is to maximize the consumption of energy produced locally. To reach this goal, based on consumption profiles of customers willing to participate in the E-cloud and given some local restrictions (e.g. wind turbines cannot be put everywhere), an optimal mix of green generation sources (in $\mathrm{kW}$ ) and local storage (in $\mathrm{kWh}$ ) needs to be computed. Then according to this computation, the required generating units and storage device are installed. A repartition mechanism grants the customer a share of the generated electricity and storage capacity. These shares are either computed offline, or dynamically adapted on line.

The project will test two models: either the DSO or a producer owns and operates the storage device.

Two flows of information (real-time for operation of the storage facility and ex-post for its settlement) are needed to correctly manage the E-Cloud and to ensure correct information exchange with the wholesale market. These information flows are completed thanks to a forecast that provides members of the E-Cloud the full capability to anticipate and obtain the maximum benefits of the local generation.

The expected benefits for the customer are a reduction of their electricity bill by a minimum of 10\%. Societal benefits should also arise: 1) easing the technical integration of renewables' generation embedded in the distribution network, and 2) avoids extra investment on the DSO network. The E-Cloud may also ensure new revenue for the DSO thanks to new services provided to the E-Cloud community.
\end{abstract}

\section{INTRODUCTION}

A microgrid is usually defined as a local network to which generators, energy storage systems, and loads are connected and which is coupled with the main grid by only one connection point. It may, under certain conditions, be disconnected from the main grid to be operated autonomously.

Besides the environmental interest (e.g. trend to circular economy), a microgrid can, for many companies, be seen as a means to avert increasing energy costs. Consequently, investing in a microgrid could be a way for these companies to hedge against higher future prices on these markets.

However, ORES and RESA, the two main DSOs in Wallonia (Belgium), think that it is possible to capture many of the advantages a microgrid can bring without being forced to build a new network, or to have only one connection point with the main grid.

This has lead them to develop the concept of an E-Cloud: an integrated power distribution network feeding an existing area of economic activity, which distributes electrical energy to industrial or commercial sites that have agreed to be part of the E-Cloud community.

The E-Cloud project is funded by the Walloon Region in the framework of the Marshall Plan 4.0 - and is coordinated by ORES. It is articulated around different work packages: test storage on the studied MV network, test and analyse the data flows and the organization of billing, analyse the regulatory impacts (tariff and market models), find the optimal techno-economic configuration in order to maximize the profits of each involved actor, provide micro and macro-economic analysis of the benefits of E-Cloud for the various market players and, finally, analyse the advantages/drawbacks of the E-Cloud concept against conventional microgrids.

Two pilot areas are foreseen, with real industrial customers, renewable energy sources and storage devices.

To have an accurate view on every aspect of this new concept, the project can count on several partners:

- Energy producers: EOLY (Colruyt Group) and Nethys Energy

- Regional development agencies (RDA): IDETA and SPI

- Universities: University of Mons (UMons) and University of Liège (ULg)

- Optimization solution provider: N-SIDE

- Distribution system operators: ORES and RESA

This paper presents the concept and the first set of collected results.

It is structured as follows. Firstly, section I defines the specific assets needed to manage an E-Cloud. Section II then characterizes the information flows and the relation 
with the wholesale market, while section III defines the roles and responsibilities of each stakeholder. Finally, sections IV and V respectively give an overview of the first results of the E-Cloud and a summary of the following steps.

\section{SECTION I: SPECIFIC E-CLOUD ASSETS}

\section{System}

The considered system is the MV network that feeds a specific economic zone (in the Walloon legislation called Zone d'Activité Economique (ZAE)).

As this network is fully integrated within the classical DSO's network (unlike pure microgrids), there is more than one connection point. Figure 1 gives an illustration of such a network.

The customers connected to this system have the choice to participate or not in the E-Cloud community. The ones that agree to participate are called members. They are indicated in yellow in Figure 1.

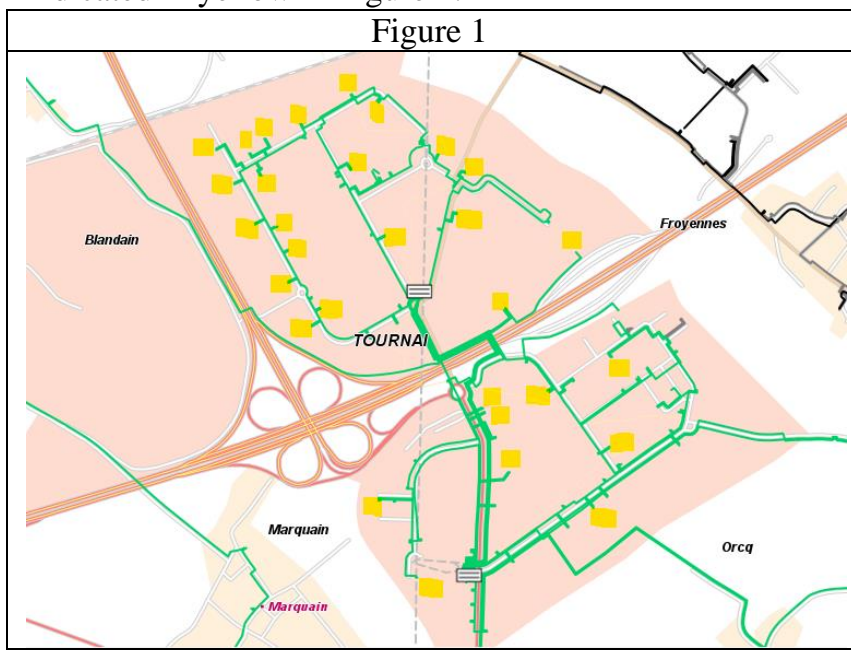

\section{Generation means}

Based on the historical profiles of the members, the goal is to design the generation means in order to minimize the Levelized Cost of Energy considering local generation and offtakes from the main grid for the whole E-Cloud community, and taking into account the following constraints:

- The investment in generation capability has to give enough financial return (Return On Investment) (subsidies included)

- Each individual member has to observe a real decrease in their electricity bill ( $\min 10 \%$ )

The considered generation capabilities to reach this optimum are renewable energy source (RES) such as photovoltaic, wind turbine ...

Of course, local constraints must be taken into account (e.g. erecting a wind turbine cannot be done everywhere).
The final results of this phase are the answers to the following questions:

- How many units of each RES generation type have to be installed, and where (e.g. on which roof), in order to obtain the optimum mix of renewable sources?

- For each of these units, what will be the installed power capacity?

When these issues are answered, each generation unit will be installed, separately metered, and the shares of generation for each member determined. These shares, in case of off-line computation, will be fixed for a long period of time (at least one year). Nevertheless, the project will assess if a change in the shares at every market period can potentially bring more benefits for a given member.

The first rule to compute these shares is a rule that allocates electricity proportionally to demand (consumption) of each member of the community:

$$
s_{j}=\frac{C_{j}}{\sum_{x=1}^{J} C_{x}}
$$

With $s_{j}$ is the share for the member $j, C_{j}$ is the consumption for the member $\mathrm{j}$ without the E-Cloud mechanism, and $\mathrm{J}$ the total of members participating to the E-Cloud community.

A second possible rule is to calculate the share based on the benefits

$$
s_{j}=\frac{G_{j}}{\sum_{x=1}^{J} G_{x}}
$$

Where $G_{j}$ is the benefit of the member $j$. There are 2 options to define this benefit. The option A is to calculate the energy consumed by the customer $j$ which comes from the local production $\left(G_{j}=E_{l, j}\right)$. The option B is equivalent to option $\mathrm{A}$ but the price of the energy is also taken into account. In this option,

$G_{j}=E_{l, j} \cdot a_{j}$ with $a_{j}$ the price at which member $j$ buys its electricity on the global market.

It seems that the most equitable method is the first one and the second one with option A as electricity locally produced is always shared according to the demand of each member without taking account of the price actually paid by each member. We considered that this price is related to their capability to negotiate their contract with their actual supplier.

\section{Storage device}

Even if the generation is optimal, there are specific times when it exceeds the consumption. Storage can be used to maximize to the use of the energy locally produced. This is also an optimization computation where the price of selling this exceeding energy to the market is an important decision variable. The optimal generation 
capabilities and optimal storage device are done in one integrated optimization problem.

If the optimization process leads to the necessity for introducing storage capacity, for the sake of simplification, this storage will be considered as one unit (Lithium Ion battery) directly connected to the MV network of the studied zone. The storage installation is also separately metered. The project partner N-SIDE is in charge of developing this investment optimization solution.

\section{SECTION II: FLOWS OF INFORMATION AND WHOLESALE MARKET VIEW}

\section{Forecast data}

Information and anticipation is a key point in order to provide each member with the maximal opportunity to capture the value of local generation. As these members are industrial customers (energy is only a means to producing their goods or services), they need time to prepare/adapt their consumption profile. For that purpose, day-ahead standard consumption profiles and forecasts of their share of RES generation will be communicated to each member. The University of Mons is developing the needed forecasting tools.

\section{Real-time data}

Real-time information (consumption and share of generation) is communicated to the consumers to help them define their balancing position (difference between their share of production and their own consumption) and, thus, to take the necessary actions to manage their demand and maximize the use of their share of production. Practically, the lack or excess of generated energy with respect to the demand of a member defines the exchanges with the storage device. However, the real exchanges with the storage device depend on the positions of the different members of the E-Cloud. Ideally, at the end of each market period, the storage device should have perfectly compensated for the deviations of the members of the E-Cloud. ORES and the University of Liège are developing the related decision (store or consume) algorithm.

\section{Ex-post data}

Generators and consumers are metered independently with a market period resolution. These metered quantities for generators are important for subsidies related to renewable generation (e.g. in Wallonia, the green certificates), and also for cross-checking the energy generated at the settlement stage.

The consumer can have direct access to their own consumption meter. With these metering devices, it is always possible to track and check the correctness of the exchanges.
The integration of real-time data into a market time step is performed and has two main purposes. The first one is to update the storage ledger (which quantity of stored energy belongs to each member). The second one is to compute the market period share of generation. The project will also have to determine how to deal with the delta between the metered consumption/generation and the integration of real-time data (e.g. considered as network losses)

\section{Market View}

Figure 2 summarizes the real-time and ex-post information flows. It can be seen that, from a market perspective, the metered consumption of a specific member is compensated by the share of generated energy for this member. This will define the actual consumption seen by the provider of a given E-Cloud member. It is the same as if this compensated consumption was measured by a virtual market meter.

\section{SECTION III: ROLES RESPONSIBILITIES IN THE E-CLOUD}

\section{Producer}

The producer invests and operates all the means of energy generation. This role can be fulfilled by the members (in an association), by an independent party or by a third-party investor. As revenue, the producer will receive a fee from the members for the installations.

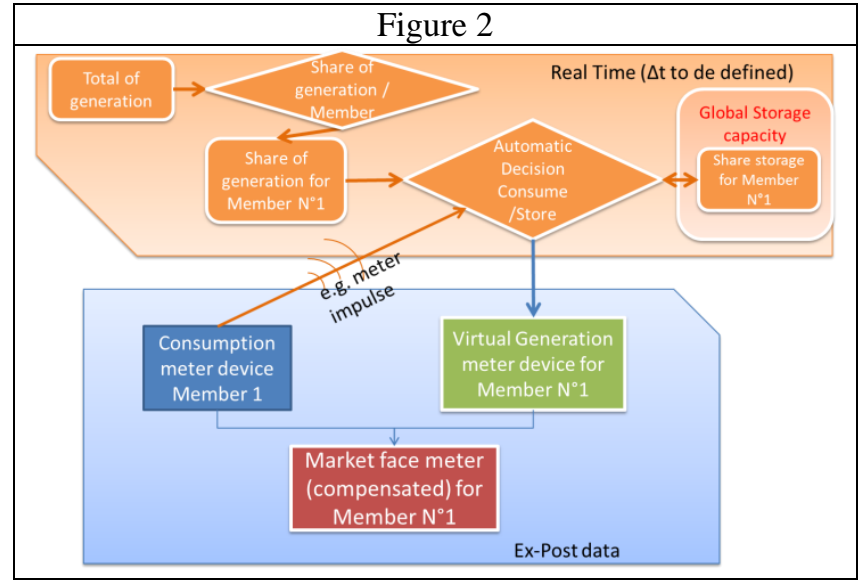

This fee can be fixed (an annuity) or can be expressed in $€ / \mathrm{MWh}$ produced. In the E-Cloud project, the annuity has been chosen and this role is done by EOLY for Pilot 1 and NETHYS for Pilot 2. For Pilot 2, the producer, NETHYS, is also the owner and the

operator of the storage facility. The producer also receives the revenue from the support mechanism for renewable generation (green certificates). 


\section{$\underline{\text { DSO }}$}

The DSOs, ORES and RESA, provide the following services:

1. Day to day management of the grid

2. Provide forecast data to each member

3. Provide real-time data in order to provide operating points to the storage unit.

4. Create and provide access to a web page for each member in order to facilitate the exchange of information

5. Calculate ex-post data and communicate the relevant information to the market

For Pilot $\mathrm{N}^{\circ} 1$, ORES also owns and operates the storage installation. The first service is covered by the traditional grid fee tariff, applied to the compensated consumptions. The services 2 to 5 (and the storage for Pilot 1) are covered by a specific tariff.

For the project, as it is foreseen in the legislation, the DSOs have to ask the regulator (CWaPE) that both pilot zones be treated as an exception and that these specific market functionalities can be implemented.

\section{Member}

The members have the freedom to 1 ) benefit solely from the local generation, 2) go further and adapt their consumption profile to get more benefits from the ECloud, and 3) choose a supplier for energy not generated within the E-Cloud (for the non-compensated energy).

\section{$\underline{\text { Regional Development Agencies (RDA) }}$}

These RDAs are the economic animators of the ZAE. Their role is to promote the concept and to organize the recruitment of consumers. The E-Cloud concept is, for them, a way to give a new dynamism to existing ZAE and to eventually attract new manufacturers and industries in these areas in order to develop the local economy.

\section{Other parties}

Members have the choice to go further than just being a passive beneficiary of local energy generation and storage: they could also adapt dynamically to this new environment. To do that, they can ask also for the help of an Energy Service Company (ESCO), which specialises in the setting up of demand-side management schemes. We note that around this E-cloud concept, ESCOs will be able to offer a new range of services.

The role of the supplier will diminish in importance with this model. Indeed, the volume of energy bough from the wholesale market will decrease with the rollout of EClouds, and the volatility of the consumption will increase due to the intermittent behaviour of the RES sources.

As a result, it may be possible that a member of this E- cloud will face an unfair commodity price from their retailer (in $€ / \mathrm{MWh}$ ) for the consumption not covered by local energy generation. Therefore, it is important that the member still has the freedom to change supplier if he/she feels that the increase in commodity price is unacceptable.

Nevertheless, a supplier can also make the choice to follow its customer by co-investing in the generation means (even fulfilling the role of producer in the ECloud) and/or propose an ESCO service.

\section{SECTION IV: FIRST RESULTS}

A first set of simulations have been carried out on several ZAEs and the results tend to confirm that, with the actual investment cost, the E-Cloud can create the requested average $10 \%$ reduction in the electricity bills of members. These promising first results need to be confirmed by the full development of the project.

Another first observation is that photovoltaic generation is the most interesting mean of RES energy, compared to wind turbines, for the E-Cloud concept. This is probably due to the fact that, in the ZAEs on which these simulations are done, a high " $8-17 \mathrm{~h}$ " working day pattern can be observed.

To conclude, storage is also an interesting factor to improve auto-consumption with a relative low energy capacity and power rating.

\section{SECTION V: NEXT STEPS}

This 40-month project started in October 2016. So far, the ZAEs for the pilot project were selected and meetings with customers are scheduled. An in-depth analysis of the regulatory context is currently being carried out. The first commissioning (generation and storage) assets are planned to start end 2017- first quarter of 2018. After that, a full year of tests will be necessary to collect enough data to accurately assess the benefits of the ECloud concept. Later on, the Universities of Mons and Liège will also carry out the macro and micro social welfare analysis, as well as establish the guidelines for further deployment of the concept in Wallonia. 